\title{
Arachnofaunistical studies in Csákányospuszta and surroundings (Tatabánya, Komárom-Esztergom county, Hungary)
}

\author{
SzITA Éva ${ }^{1}$, Kovács PÉTER ${ }^{2} \&$ SzINETÁr CsABA ${ }^{3}$
}

\begin{abstract}
1Plant Protection Institute, Centre for Agricultural Research, Eövös Loránd Reserch Network, 1022 Budapest Herman Ottó út 15., e-mail: szita.eva@atk.hu

2Szombathelyi Arachnológiai Mühely, 9700 Szombathely Károlyi Gáspár tér 4., e-mail: kovacsp@locart.hu ${ }^{3}$ ELTE SEK Biológia Tanszék, 9700 Szombathely Károlyi Gáspár tér 4., e-mail: szcsaba.bdtf@gmail.com
\end{abstract}

Szita, É., Kovács, P. \& Szinetár, Cs.: Arachnofaunistical studies in Csákányospuszta and surroundings (Tatabánya, Komárom-Esztergom county, Hungary).

Abstract: Faunistical investigations of the 17th Biodiversity Days revealed the occurrence of 129 spider (Araneae) species in Csákányospuszta, northernmost part of Vértes Mountains, Hungary. Among the collected species there was 1 proteceted species (Atypus piceus (Sulzer, 1776)) and were several rare ones to the Hungarian fauna (Collinsia inerrans (O. P.-Cambridge, 1885), Entelecara flavipes (Blackwall, 1834), Metopobactrus prominulus (O. P.-Cambridge, 1872), Zora parallela Simon, 1878, Lasaeola prona (Menge, 1868), Tmarus stellio Simon, 1875).

Keywords: biodiversity days, spiders, Vértes Mountains, Duna-Ipoly National Park

\section{Introduction}

The 17th Biodiversity Days (BD17), a two-day intensive faunistic and floristic survey was conducted in Csákányospuszta, at the nothernmost part of Vértes Mountains (DunaIpoly National Park, Hungary) in 2017.

Arachno-faunistical research has a long tradition in Hungary. At the end of the 19th century, Kornél Chyzer and Ladislaw Kulczynski performed a large-scale collecting from the pre-World War I territory of Hungary. Based on their study, the presence of 32 spider species was detected from Komárom-Esztergom county (CHYZER \& KULCZYNSKI 1891, 1894, 1897). This list was updated by CHYZER (1909) with one species (Eresus kollari Rossi, 1846) and then by MikHailov \& SzInetár (1997) with two more species. So far, 35 species have been known from Komárom-Esztergom county from the following places: Esztergom (18 species), Felsőgalla (cave) (7 species), Hajagos-Turul-hegy, Gerecse (2 species), Peskő, Gerecse (1 species), Pilismarót (14 species), Tata (14 species). Two of these collecting places, Hajagos-Turul-hegy and Felsögalla (cave), belong to the administrative territory of Tatabánya. These data were not added to the results of the BD17 spider checklist, but species overlapping with the previous county species list were marked in the current BD17 checklist. 
Arachnological research has recently been carried out in two areas close to the southern border of Komárom-Esztergom county (EICHARDT et al. 2018, EICHARDT et al. 2020). In the future, it is worth considering these studies for the distribution of the species. Natural geographical boundaries are inherently more important than administrative (county) boundaries.

\section{Material and methods}

The 17th Biodiversity Days were held on June 9-11, 2017 in Csákányospuszta (Tatabánya), organised by the Hungarian Biodiversity Research Society. The designated core area comprises a variety of habitat types (Fig. 1, Table 1), and further extensions were also added to the study area, as a south-western dolomite rock grassland, Hosszú Hill near Szárliget, and two wetlands near Síkvölgypuszta (Tatabánya) and Vértessomló, as well as the northern part of Tatabánya with the Selim Cave belonging to Gerecse (the latter is not shown in Fig. 1).

Several methods were used to explore the spider fauna: individual spider collecting, sweep net, limb beating, hand-held suction sampling (D-vac), and pitfall trap. The following colleagues took part in collecting of spiders: Elöd Kondorosy, Csaba Kutasi, Péter Kovács, Tibor Kovács, János Novák, Csaba Szinetár, Éva Szita. The collecting methods and collector names used at each sampling area are shown in Table 1. Spiders were conserved in 70\% ethanol, and adult individuals were identified by NENTWIG et al. (2021). The nomenclature of species and families follows the current version of the World Spider Catalog (2021).

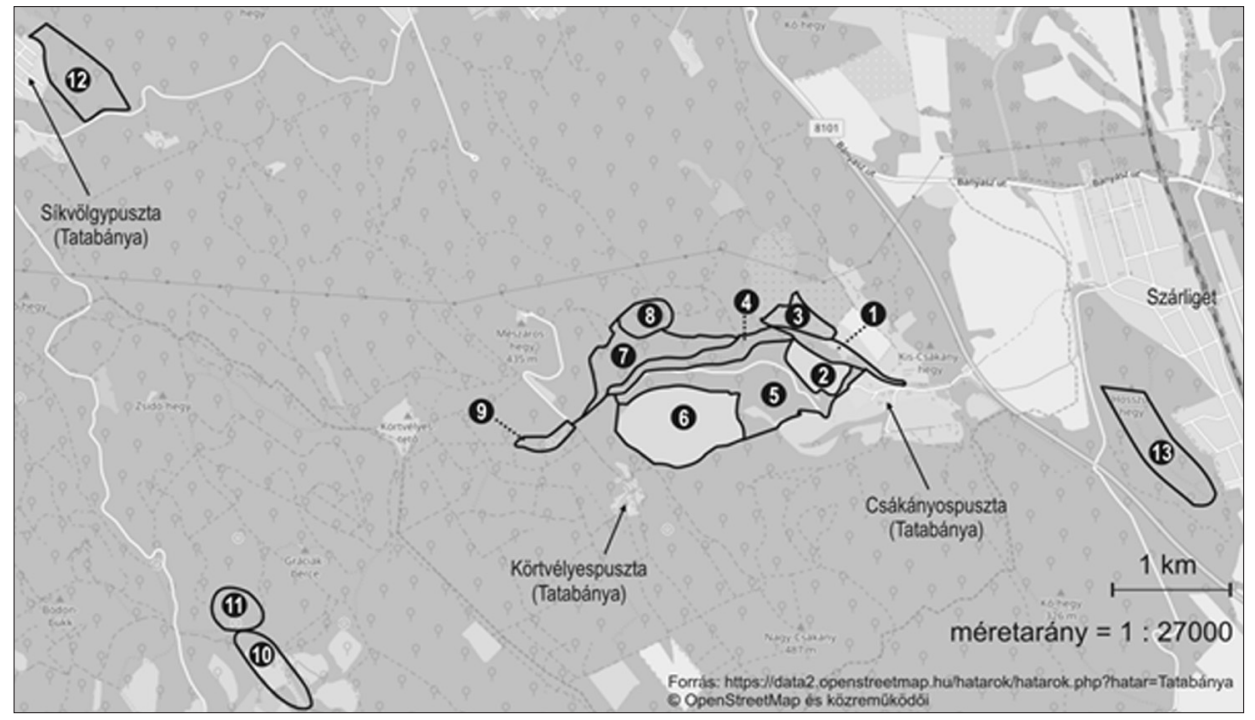

Fig. 1: Sampling sites in and around Csákányospuszta in the 17th Biodiversity Days See the meaning of the numbers in Table 1. 
Table 1: Data of sampling sites

Abbreviation of collector names: KE - Kondorosy, Elöd; KCs - Kutasi, Csaba; KP - Kovács, Péter; KT Kovács, Tibor; NJ - Novák, János; SzCs - Szinetár, Csaba; SzÉ - Szita, Éva

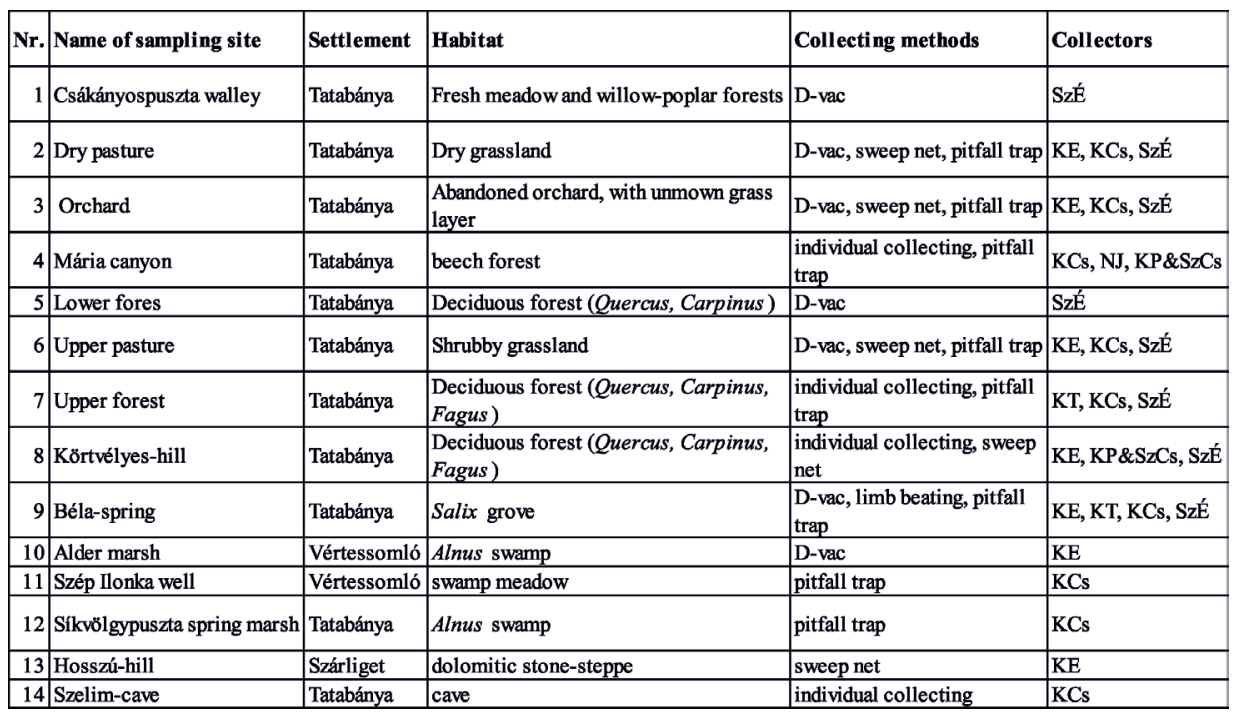

\section{Results and discussion}

A total of 672 spider specimens (552 adult) were collected during the two collecting days of the BD17, belonging to 129 species of 26 spider families (Table 2). The most species-rich spider families proved to be: Linyphiidae ( 24 species), Theridiidae (17 species), Lycosidae (12 species), Thomisidae (12 species), Gnaphosidae (9 species). Information on the frequency of a species in Hungary and the naturalness of its habitat is provided for all species in Table 2 .

Our results revealed the presence of one protected species (Atypus piceus (Sulzer, 1776)) and several rare species (Collinsia inerrans (OP-Cambridge, 1885), Entelecara flavipes (Blackwall, 1834), Metopobactrus prominulus (OP-Cambridge, 1872), Zora parallela Simon, 1878, Lasaeola prona (Menge, 1868), Tmarus stellio Simon, 1875). The species that are discussed separately below due to their rarity or protection were marked as underlined. Table 2 provides information on abundence of species and originality of their habitat, adapted to Hungary based on BUCHAR \& RŮŽIČKA (2002).

Csákányospuszta and its surroundings, chosen as the location for the 17th Biodiversity Days, have a fairly large habitat heterogenity. This fact is well reflected in the number of spider species caught (129), which is the second most species-rich collection compared to previous years of Biodiversity Days (SzINETÁR et al. 2016). The vast majority of the species were detected in natural and semi-natural habitats. Species associated with artificial habitats were found in the guest house of Csákányospuszta (Parasteatoda tepidariorum) and in the Szelim Cave (Tegenaria domestica). 20 species of the species on our list overlap with the species previously detected from Komárom-Esztergom county, thus the occurrence of 155 spider species have been confirmed from the county currently. 


\section{Rare and protected species worth for attention}

\section{Atypus piceus (Sulzer, 1776)}

This is the only protected species among the spiders detected. A. piceus occurres in a wide range of natural and semi-natural woody areas. Adult males switch to a vagabond lifestyle, thus these specimens are most in sight, or trapped in pitfall traps. Two male specimens were caught in pitfall traps at sampling site Nr. 2 (Dry pasture)

Collinsia inerrans (O. P.-Cambridge, 1885)

Palaearctic species, typical for open grassy habitats. Expansion of its distribution area has been observed in the recent decades (NENTwIG et al. 2021), and its current data also supports this. Two female specimens were found at sampling site Nr. 2 (Dry pasture).

Entelecara flavipes (Blackwall, 1834)

Rare linyphiid spider with palaearctic distribution. Predominantly occurs in forests with low vegetation (NENTwig et al. 2021).

Metopobactrus prominulus (O. P.-Cambridge, 1872)

A rare linyphiid spider with Holarctic distribution, living on the ground level. It was first reported in Hungary from the wetlands of Upper Tisza Region (LoKsA 1981). Further occurrence data are from Gyürüfü, Transdanubia (Kovícs et al. 2009) and sand steppe of Kisalföld (SzINETÁr et al. 2015). In Csákányospuszta, four females were found at sampling site Nr. 3 (Orchard).

\section{Zora parallela Simon, 1878}

This extremely rare spider species is known from Europe and some parts of Asia. We have only one published data from Hungary so far. It was collected in Gyürüfü (1st Hungarian Biodiversity Days) by motorised hand-held suction sampling (D-vac) in 2007 (Kovícs et al. 2009). In this study, 3 specimens were recorded in grassland habitats at sampling sites Nr. 2 (Dry pasture) and Nr. 6 (Upper pasture), respectively.

\section{Lasaeola prona (Menge, 1868)}

Rarely collected theridiid spiders with holarctic distribution. Species associated with sunny forest edges. It was first recorded in Hungary from Örség (SzINETÁr 1995). It was recently collected in apple orchards in the Trans-Tisza region (MEzöFI \& MARKó 2018). In Csákányospuszta, a male specimen was found in shrubby grassland.

\section{Tmarus stellio Simon, 1875}

This crab spider species was recorded from Southern Europe to Japan. It is much rarer, than Tmarus piger, which is common in Hungary. Although it was already included in the monograph published at the beginning of the last century (CHYZER \& KULCZYNSKI 1891), from Tokaj and Sátoraljaújhely, our knowledge is quite limited about this species. Like its relative, it lives on the branches of trees. A male specimen was collected at sampling site $\mathrm{Nr} 3$ (Orchard). 
Table 2. List of spiders collected in Csákányospuszta and surroundings

*Occurrence of a species in Komárom-Esztergom county was reported in previous literatures. Underlined species names are discussed separately in the text. Codes of study sites see in Table 1. Abbreviations. Abundance: $\mathrm{R}$ - rare, MF - moderately frequent, $\mathrm{F}$ - frequent; Originality of habitat: $\mathrm{N}$ - natural, $\mathrm{SN}$ - semi-natural, $\mathrm{D}$ disturbed, $\mathrm{A}-$ artificial

\begin{tabular}{|c|c|c|c|}
\hline Taxon (family and species name) & $\begin{array}{l}\text { Sampling site } \\
\text { code }\end{array}$ & Abundance & $\begin{array}{l}\text { Originality } \\
\text { of habitat }\end{array}$ \\
\hline \multicolumn{4}{|l|}{ Agelenidae } \\
\hline Histopona torpida (C. L. Koch, 1837) & $4,5,6,7,11$ & MF & $\mathrm{N}, \mathrm{SN}$ \\
\hline Inermocoelotes inermis (L. Koch, 1855) & 4,6 & MF & $\mathrm{N}, \mathrm{SN}$ \\
\hline *Tegenaria domestica (Clerck, 1757) & 14 & $\mathrm{~F}$ & A \\
\hline Tegenaria ferruginea (Panzer, 1804) & 4 & $\mathrm{~F}$ & $\mathrm{~N}, \mathrm{SN}, \mathrm{A}$ \\
\hline Tegenaria silvestris L. Koch, 1872 & 5 & $\mathrm{~F}$ & $\mathrm{~N}, \mathrm{SN}$ \\
\hline \multicolumn{4}{|l|}{ Amaurobiidae } \\
\hline${ }^{*}$ Amaurobius ferox (Walckenaer, 1830) & 8 & $\mathrm{~F}$ & $\mathrm{SN}, \mathrm{D}, \mathrm{A}$ \\
\hline \multicolumn{4}{|l|}{ Anyphaenidae } \\
\hline Anyphaena accentuata (Walckenaer, 1802) & 8,9 & $\mathrm{~F}$ & $\mathrm{~N}, \mathrm{SN}$ \\
\hline \multicolumn{4}{|l|}{ Araneidae } \\
\hline Araniella opisthographa (Kulczynski, 1905) & $3,4,8,9$ & $\mathrm{~F}$ & $\mathrm{~N}, \mathrm{SN}$ \\
\hline Hypsosinga pygmaea (Sundevall, 1832) & 10 & MF & $\mathrm{N}, \mathrm{SN}$ \\
\hline Hypsosinga sanguinea (C. L. Koch, 1844) & 2 & MF & $\mathrm{N}, \mathrm{SN}$ \\
\hline Mangora acalypha (Walckenaer, 1802) & $2,3,6,8,13$ & $\mathrm{~F}$ & $\mathrm{~N}, \mathrm{SN}, \mathrm{D}$ \\
\hline Zilla dioida (Walckenaer, 1802) & 8 & $\mathrm{~F}$ & $\mathrm{~N}, \mathrm{SN}$ \\
\hline \multicolumn{4}{|l|}{\begin{tabular}{|l|l} 
Atypidae \\
\end{tabular}} \\
\hline Atypus piceus (Sulzer, 1776) & 2 & MF & $\mathrm{N}$ \\
\hline \multicolumn{4}{|l|}{ Cheiracanthidae } \\
\hline Cheiracanthium elegans Thorell, 1875 & 8 & MF & $\mathrm{N}$ \\
\hline \multicolumn{4}{|l|}{ Clubionidae } \\
\hline Clubiona diversa O. P.-Cambridge, 1862 & 6 & MF & $\mathrm{N}$ \\
\hline Clubiona neglecta O. P.-Cambridge, 1862 & 2,6 & MF? & $\mathrm{N}, \mathrm{SN}$ \\
\hline Clubiona terrestris Westring, 1851 & 9 & MF & $\mathrm{N}, \mathrm{SN}$ \\
\hline Clubiona trivialis C. L. Koch, 1843 & 2 & MF & $\mathrm{N}, \mathrm{SN}$ \\
\hline \multicolumn{4}{|l|}{ Dictynidae } \\
\hline Argenna subnigra (O. P.-Cambridge, 1861) & 2,6 & MF & $\mathrm{N}, \mathrm{SN}$ \\
\hline Brigittea latens (Fabricius, 1775) & 4,6 & MF & $\mathrm{N}, \mathrm{SN}$ \\
\hline Dictyna arundinacea (Linnaeus, 1758) & 3 & $\mathrm{~F}$ & $\mathrm{~N}, \mathrm{SN}, \mathrm{D}$ \\
\hline *Dictyna uncinata Thorell, 1856 & 4 & MF & N, SN, D \\
\hline Lathys humilis (Blackwall, 1855) & 2 & MF & $\mathrm{N}, \mathrm{SN}$ \\
\hline Nigma flavescens (Walckenaer, 1830) & 4,5 & MF & $\mathrm{N}, \mathrm{SN}$ \\
\hline \multicolumn{4}{|l|}{ Dysderidae } \\
\hline *Harpactea hombergi (Scopoli, 1763) & 4 & MF & $\mathrm{N}, \mathrm{SN}$ \\
\hline Harpactea rubicunda (C. L. Koch, 1838) & 4 & $\mathrm{~F}$ & $\mathrm{~N}, \mathrm{SN}, \mathrm{A}$ \\
\hline \multicolumn{4}{|l|}{ Gnaphosidae } \\
\hline Drassodes lapidosus (Walckenaer, 1802) & 7 & $\mathrm{~F}$ & $\mathrm{~N}, \mathrm{SN}$ \\
\hline Drassyllus praeficus (L. Koch, 1866) & 3 & MF & $\mathrm{N}, \mathrm{SN}$ \\
\hline Drassyllus pumilus (C. L. Koch, 1839) & 2,7 & MF & $\mathrm{N}$ \\
\hline Drassyllus villicus (Thorell, 1875) & 2 & $\mathrm{~F}$ & $\mathrm{~N}$ \\
\hline Haplodrassus silvestris (Blackwall, 1833) & 7,12 & $\mathrm{~F}$ & $\mathrm{~N}, \mathrm{SN}$ \\
\hline Micaria dives (Lucas, 1846) & 3 & MF & $\mathrm{N},(\mathrm{SN})$ \\
\hline Micaria formicaria (Sundevall, 1832) & 3 & MF & $\mathrm{N}, \mathrm{SN}$ \\
\hline Trachyzelotes pedestris (C. L. Koch, 1837) & 7 & $\mathrm{~F}$ & $\mathrm{~N}, \mathrm{SN},(\mathrm{D})$ \\
\hline Zelotes subterranus (C. L. Koch, 1833) & 11 & $\mathrm{~F}$ & $\mathrm{~N}, \mathrm{SN}, \mathrm{D}$ \\
\hline
\end{tabular}


Table $2 / 2$ continuation

\begin{tabular}{|c|c|c|c|}
\hline Taxon (family and species name) & $\begin{array}{l}\text { Sampling site } \\
\text { code }\end{array}$ & Abundance & $\begin{array}{l}\text { Originality } \\
\text { of habitat }\end{array}$ \\
\hline \multicolumn{4}{|l|}{ Hahniidae } \\
\hline Cicurina cicur (Fabricius, 1793) & 6 & MF & $\mathrm{N}, \mathrm{SN}, \mathrm{D}$ \\
\hline Hahnia nava (Blackwall, 1841) & 2 & $\mathrm{~F}$ & $\mathrm{~N}, \mathrm{SN}$ \\
\hline \multicolumn{4}{|l|}{ Linyphiidae } \\
\hline Agyneta affinis (Kulczynski, 1898) & 2,6 & $\mathbf{R}$ & $\mathrm{N}, \mathrm{SN}$ \\
\hline Agyneta rurestris (C. L. Koch, 1836) & 6,10 & $\mathrm{~F}$ & $\mathrm{~N}, \mathrm{SN}, \mathrm{D}$ \\
\hline Agyneta simplicitarsis (Simon, 1884) & 2,3 & $\mathrm{~F}$ & $\mathrm{~N}, \mathrm{SN}$ \\
\hline Bathyphantes nigrinus (Westring, 1851) & 9 & $\mathrm{~F}$ & $\mathrm{~N}, \mathrm{SN}$ \\
\hline Ceratinella brevipes (Westring, 1851) & 7 & $\mathrm{~F}$ & $\mathrm{~N}, \mathrm{SN}$ \\
\hline Collinsia inerrans (O. P.-Cambridge, 1885) & 2 & $\mathbf{R}$ & $\mathrm{N}, \mathrm{SN}(?)$ \\
\hline Diplostyla concolor (Wider, 1834) & 7,8 & $\mathrm{~F}$ & $\mathrm{~N}, \mathrm{SN}, \mathrm{D}$ \\
\hline Entelecara flavipes (Blackwall, 1834) & 6 & $\mathbf{R}$ & $\mathrm{N}, \mathrm{SN}$ \\
\hline Erigone dentipalpis (Wider, 1834) & 1,3 & $\mathrm{~F}$ & $\mathrm{~N}, \mathrm{SN}, \mathrm{D}$ \\
\hline Metopobactrus prominulus (O. P.-Cambridge, 1872) & 3 & $\mathbf{R}$ & $\mathrm{N}, \mathrm{SN}$ \\
\hline Micrargus herbigradus (Blackwall, 1854) & 2,5 & $\mathrm{~F}$ & $\mathrm{~N}, \mathrm{SN}$ \\
\hline Minicia marginella (Wider, 1834) & 6 & $\mathrm{MF}$ & $\mathrm{N}, \mathrm{SN}$ \\
\hline Neriene clathrata (Sundevall, 1830) & 2,3 & $\mathrm{~F}$ & $\mathrm{~N}, \mathrm{SN}$ \\
\hline Neriene furtiva (O. P.-Cambridge, 1870) & 3 & $\mathbf{R}$ & $\mathrm{N}$ \\
\hline Neriene radiata (Walckenaer, 1841) & 4 & $\mathrm{~F}$ & $\mathrm{~N}, \mathrm{SN}$ \\
\hline Oedothorax agrestis (Blackwall, 1853) & 9 & MF & $\mathrm{N}, \mathrm{SN}$ \\
\hline Oedothorax apicatus (Blackwall, 1850) & 1,3 & $\mathrm{~F}$ & $\mathrm{~N}, \mathrm{SN}, \mathrm{D}$ \\
\hline Oedothorax fuscus (Blackwall, 1834) & 9 & MF & $\mathrm{N}, \mathrm{SN}, \mathrm{D}$ \\
\hline Pocadicnemis pumila (Blackwall, 1841) & 6 & $\mathrm{~F}$ & $\mathrm{~N}, \mathrm{SN}$ \\
\hline Tenuiphantes flavipes (Blackwall, 1854) & 4,5 & $\mathrm{~F}$ & $\mathrm{~N}, \mathrm{SN}$ \\
\hline Tenuiphantes tenuis (Blackwall, 1852) & 10 & $\mathrm{~F}$ & $\mathrm{~N}, \mathrm{SN}, \mathrm{D}$ \\
\hline Trichoncus hackmani Millidge, 1956 & $1,2,3,13$ & $\mathbf{R}$ & $\mathrm{N}, \mathrm{SN}$ \\
\hline Walckenaeri atrotibialis (O. P.-Cambridge, 1878) & 4,11 & MF & $\mathrm{N}, \mathrm{SN}$ \\
\hline \multicolumn{4}{|l|}{ Liocranidae } \\
\hline Agroeca brunnea (Blackwall, 1833) & $6,9,11$ & $\mathrm{~F}$ & $\mathrm{~N}, \mathrm{SN}$ \\
\hline \multicolumn{4}{|l|}{ Lycosidae } \\
\hline Alopecosa cuneata (Clerck, 1757) & 2 & $\mathrm{~F}$ & $\mathrm{~N}, \mathrm{SN}, \mathrm{D}$ \\
\hline Alopecosa pulverulenta (Clerck, 1757) & 2 & $\mathrm{~F}$ & $\mathrm{~N}, \mathrm{SN}, \mathrm{D}$ \\
\hline Alopecosa sulzeri (Pavesi, 1873) & 7 & MF & $\mathrm{N}$ \\
\hline Aulonia albimana (Walckenaer, 1805) & 2,6 & $\mathrm{~F}$ & $\mathrm{~N}, \mathrm{SN},(\mathrm{D})$ \\
\hline Pardosa alacris (C. L. Koch, 1833) & $7,9,11,12$ & $\mathrm{~F}$ & $\mathrm{~N}, \mathrm{SN}$ \\
\hline Pardosa amentata (Clerck, 1757) & 9,11 & $\mathrm{~F}$ & $\mathrm{~N}, \mathrm{SN}, \mathrm{D}$ \\
\hline Pardosa bifasciata (C. L. Koch, 1834) & 3,6 & MF & $\mathrm{N}$ \\
\hline${ }^{*}$ Pardosa lugubris (Walckenaer, 1802) & 11 & $\mathrm{~F}$ & $\mathrm{~N}, \mathrm{SN}, \mathrm{D}$ \\
\hline Pardosa riparia (C. L. Koch, 1833) & $2,6,7,11$ & MF & $\mathrm{N}, \mathrm{SN}$ \\
\hline Piratula hygrophila (Thorell, 1872) & 11,12 & $\mathrm{~F}$ & $\mathrm{~N}, \mathrm{SN}$ \\
\hline Trochosa terricola Thorell, 1856 & $1,7,11$ & $\mathrm{~F}$ & $\mathrm{~N}, \mathrm{SN}, \mathrm{D}$ \\
\hline Xerolycosa miniata (C. L. Koch, 1834) & 1 & $\mathrm{~F}$ & $\mathrm{~N}, \mathrm{SN}$ \\
\hline \multicolumn{4}{|l|}{ Mimetidae } \\
\hline *Ero aphana (Walckenaer, 1802) & 2 & $\mathrm{~F}(\mathrm{MF})$ & $\mathbf{N}$ \\
\hline \multicolumn{4}{|l|}{ Miturgidae } \\
\hline Zora parallela Simon, 1878 & 2,6 & $\mathbf{R}$ & $\mathrm{N}, \mathrm{SN}$ \\
\hline Zora pardalis Simon, 1878 & 6 & MF & $(\mathrm{SN})$ \\
\hline Zora spinimana Simon, 1878 & 2,11 & $\mathrm{~F}$ & $\mathrm{~N}, \mathrm{SN}, \mathrm{D}$ \\
\hline
\end{tabular}


Table $2 / 3$ continuation

\begin{tabular}{|c|c|c|c|}
\hline Taxon (family and species name) & \begin{tabular}{|l|}
$\begin{array}{l}\text { Sampling site } \\
\text { code }\end{array}$ \\
\end{tabular} & Abundance & \begin{tabular}{|l|}
$\begin{array}{l}\text { Originality } \\
\text { of habitat }\end{array}$ \\
\end{tabular} \\
\hline \multicolumn{4}{|l|}{ Mysmenidae } \\
\hline Microdipoena jobi (Kraus, 1967) & 6 & $\mathrm{R},(\mathrm{MF})$ & $\mathrm{N}, \mathrm{SN}$ \\
\hline \multicolumn{4}{|l|}{ Oxyopidae } \\
\hline Oxyopes lineatus Latreille, 1806 & 3 & $\mathrm{R},(\mathrm{MF})$ & $\mathrm{N}, \mathrm{SN}$ \\
\hline \multicolumn{4}{|l|}{ Philodromidae } \\
\hline Philodromus albidus Kulczynski, 1911 & 9 & (MF)? & $\mathrm{N}, \mathrm{SN}, \mathrm{D}$ \\
\hline Philodromus aureolus (Clerck, 1757) & 13 & $\mathrm{~F}$ & $\mathrm{~N}, \mathrm{SN}, \mathrm{D}$ \\
\hline Thanatus arenarius L. Koch, 1872 & 3 & MF & $\mathrm{N}$ \\
\hline Tibellus oblongus (Walckenaer, 1802) & 6,13 & $\mathrm{~F}$ & $\mathrm{~N}, \mathrm{SN}, \mathrm{D}$ \\
\hline \multicolumn{4}{|l|}{ Salticidae } \\
\hline *Ballus chalybeius (Walckenaer, 1802) & $6,8,9$ & MF & $\mathrm{N}, \mathrm{SN}$ \\
\hline Euophrys frontalis (Walckenaer, 1802) & 6 & $\mathrm{~F}$ & $\mathrm{~N}, \mathrm{SN}$ \\
\hline Euophrys herbigrada (Simon, 1871) & 1 & $\mathbf{R}$ & $\mathrm{N}$ \\
\hline Evarcha arcuata (Clerck, 1757) & 3,6 & $\mathrm{~F}$ & $\mathrm{~N}, \mathrm{SN}$ \\
\hline *Evarcha falcata (Clerck, 1757) & 3,13 & $\mathrm{~F}$ & $\mathrm{~N}, \mathrm{SN}$ \\
\hline Evarcha laetabunda (C. L. Koch, 1846) & $3,8,13$ & $\mathrm{~F}$ & $\mathrm{~N}$ \\
\hline Heliophanus auratus C. L. Koch, 1835 & 2,6 & MF & $\mathrm{N}, \mathrm{SN}$ \\
\hline *Heliophanus cupreus (Walckenaer, 1802) & $3,8,13$ & $\mathrm{~F}$ & $\mathrm{~N}, \mathrm{SN}$ \\
\hline Heliophanus flavipes (Hahn, 1832) & 3,13 & $\mathrm{~F}$ & $\mathrm{~N}$ \\
\hline *Macaroeris nidicolens (Walckenaer, 1802) & 1,8 & MF & $\mathrm{N}, \mathrm{SN}$ \\
\hline *Salticus zebraneus (C. L. Koch, 1837) & 13 & MF & $\mathrm{N}, \mathrm{SN}$ \\
\hline \multicolumn{4}{|l|}{ Segestriidae } \\
\hline Segestria bavariaca C. L. Koch, 1843 & 4 & MF & $\mathrm{N}, \mathrm{SN}$ \\
\hline Segestria senoculata (Linnaeus, 1758) & 4 & MF & $\mathrm{N}, \mathrm{SN}$ \\
\hline \multicolumn{4}{|l|}{ Tetragnathidae } \\
\hline Metellina segmentata (Clerck, 1757) & 4 & F & $\mathrm{N}, \mathrm{SN}, \mathrm{D}$ \\
\hline *Pachygnatha degeeri Sundevall, 1830 & $2,11,12$ & $\mathrm{~F}$ & $\mathrm{~N}, \mathrm{SN}, \mathrm{D}$ \\
\hline \multicolumn{4}{|l|}{ Theridiidae } \\
\hline Asagena phalerata (Panzer, 1801) & 7 & $\mathrm{~F}$ & $\mathrm{~N}, \mathrm{SN}$ \\
\hline Enoplognatha mordax (Thorell, 1875) & 6,8 & $\mathbf{R}$ & $\mathrm{N}$ \\
\hline *Enoplognatha ovata (Clerck, 1757) & 4,9 & $\mathrm{~F}$ & $\mathrm{~N}, \mathrm{SN}, \mathrm{D}$ \\
\hline Enoplognatha thoracica (Hahn, 1833) & 3 & $\mathrm{~F}$ & $\mathrm{~N}, \mathrm{SN}, \mathrm{D}$ \\
\hline Episinus truncatus Latreille, 1809 & 5 & $\mathrm{~F}$ & $\mathrm{~N}, \mathrm{SN}$ \\
\hline Heterotheridion nigrovariegatum (Simon, 1873) & 8 & $\mathrm{R}$ & $\mathrm{N}, \mathrm{SN}$ \\
\hline Lasaeola prona (Menge, 1868) & 6 & $\mathrm{R}$ & $\mathrm{N}$ \\
\hline Laseola tristis (Hahn, 1833) & 4 & $\mathbf{R}$ & $\mathrm{N}, \mathrm{SN}$ \\
\hline Neottiura bimaculata (Linnaeus, 1767) & $3,4,6,8,13$ & $\mathrm{~F}$ & $\mathrm{~N}, \mathrm{SN}, \mathrm{D}$ \\
\hline Neottiura suaveolens (Simon, 1879) & 2,6 & MF & $\mathrm{N}, \mathrm{SN}$ \\
\hline *Parasteatoda tepidariorum (C. L. Koch, 1841) & 7 & $\mathrm{~F}$ & $\mathrm{~A}$ \\
\hline Phylloneta impressa (L. Koch, 1881) & $3,6,13$ & $\mathrm{~F}$ & $\mathrm{~N}, \mathrm{SN}, \mathrm{D}$ \\
\hline *Platnickina tincta (Walckenaer, 1802) & $2,3,6,8,13$ & $\mathrm{~F}$ & $\mathrm{~N}, \mathrm{SN}$ \\
\hline Robertus arundineti (O. P.-Cambridge, 1871) & $5,6,8$ & $\mathrm{~F}$ & $\mathrm{~N}, \mathrm{SN}, \mathrm{D}$ \\
\hline Robertus lividus (Blackwall, 1836) & $4,5,8$ & $\mathrm{~F}$ & $\mathrm{~N}, \mathrm{SN}$ \\
\hline *Steatoda bipunctata (Linnaeus, 1758) & 7 & $\mathrm{~F}$ & $\mathrm{~N}, \mathrm{SN}, \mathrm{A}$ \\
\hline
\end{tabular}


Table $2 / 4$ continuation

\begin{tabular}{|c|c|c|c|}
\hline Taxon (family and species name) & \begin{tabular}{|l} 
Sampling site \\
code
\end{tabular} & Abundance & $\begin{array}{l}\text { Originality } \\
\text { of habitat }\end{array}$ \\
\hline Theridion varians Hahn, 1833 & 5 & $\mathrm{~F}$ & $\mathrm{~N}, \mathrm{SN}, \mathrm{A}$ \\
\hline \multicolumn{4}{|l|}{ Thomisidae } \\
\hline *Ebrechtella tricuspidata (Fabricius, 1775) & $3,4,6,13$ & $\mathrm{~F}$ & $\mathrm{~N}, \mathrm{SN}$ \\
\hline Misumena vatia (Clerck, 1757) & 6,9 & $\mathrm{~F}$ & $\mathrm{~N}, \mathrm{SN}$ \\
\hline Ozyptila claveata (Walckenaer, 1837) & 1 & MF & $\mathrm{N}$ \\
\hline *Ozyptila praticola (C. L. Koch, 1837) & 4,11 & $\mathrm{~F}$ & $\mathrm{~N}, \mathrm{SN}$ \\
\hline Ozyptila simplex (O. P.-Cambridge, 1862) & 13 & MF & $\mathrm{N}, \mathrm{SN}$ \\
\hline *Synema globosum (Fabricius, 1775) & 9 & $\mathrm{~F}$ & $\mathrm{~N}, \mathrm{SN}$ \\
\hline Synema ornatum (Thorell, 1875) & 6,8 & & \\
\hline *Thomisus onustus Walckenaer, 1806 & 2,13 & F & N, SN, D \\
\hline Tmarus stellio Simon, 1875 & 3 & $\mathbf{R}$ & $\mathrm{N}$ \\
\hline Xysticus erraticus (Blackwall, 1834) & 2 & $\mathrm{~F}$ & $\mathrm{~N}, \mathrm{SN}$ \\
\hline Xysticus kochi Thorell, 1872 & 13 & $\mathrm{~F}$ & $\mathrm{~N}, \mathrm{SN}, \mathrm{D}$ \\
\hline Xysticus luctator L. Koch, 1870 & 7,11 & MF? & $\mathrm{N}, \mathrm{SN}$ \\
\hline \multicolumn{4}{|l|}{ Titanoecidae } \\
\hline Titanoeca schineri L. Koch, 1872 & 3,7 & $\mathrm{~F}$ & $\mathrm{~N}, \mathrm{SN}$ \\
\hline \multicolumn{4}{|l|}{ Uloboridae } \\
\hline Uloborus walckenaerius Latreille, 1806 & 3,13 & MF & $\mathrm{N}$ \\
\hline
\end{tabular}

\section{Acknowledgments}

The authors wish to thank Tibor Kovács and the Hungarian Biodiversity Research Society for organizing the event and the territorially competent staff of the Duna-Ipoly National Park Directorate for their help in fieldworks. Thanks are due to the following colleagues for their participation in collectings: Elöd Kondorosy, Csaba Kutasi, János Novák. 


\section{References}

Buchar, J. \& RŮŽIČKa, V. 2002: Catalogue of spiders of the Czech Republic. - Peres Publishers, Praha, pp.

Catalog W. S. World Spider Catalog. - Version 22.0. Natural History Museum Bern, online at http://wsc. nmbe.ch, accessed on \{date of access 01.03.2021\}. doi: 10.24436/2 http://wsc.nmbe.ch

CHyzer, K. 1909: Az Eresus niger előfordulása hazánkban. - Természettudományi Közlemények 41: 296.

Chyzer, K. \& Kulczynski, L. 1891: Araneae Hungariae. Tomus I: Salticoidae, Oxyopoidae, Lycosoidae, Heteropodoidae, Misumenoidae, Euetrioidae, Tetragnathoidae, Uloboroidae, Pholcoidae, Scytodoidae, Urocteoidae, Eresoidae, Dictynoidae. - Academie Scientiarum Hungaricae, Budapest, 170 pp.

Chyzer, K. \& Kulczynski, L. 1894: Araneae Hungariae. Tomus II, pars prior : Theridioidae. - Academie Scientiarum Hungaricae, Budapest, $151 \mathrm{pp}$.

Chyzer, K. \& Kulczynski, L. 1897: Araneae Hungariae. Tomus II. pars posterior: Zodarioidae, Agalenoidae, Drassoidae, Zoropseoidae, Dysderoidae, Filistatoidae, Calommatoidae, Theraphosoidae. - Academie Scientiarum Hungaricae, Budapest, 147-366 pp.

Eichardt, J., Egessy, I. P., LÉvaI, G. \& TímÁr, M. 2018: A Nagy-tiszta (Gánt, Észak-Vértes) talajfelszíni pókfaunájának (Araneae) vizsgálata 2008-2014 között. - Folia Musei historico-naturalis Bakonyiensis 35 : 113-129.

Eichardt, J., Kutasi, Cs. \& Szinetár, Cs. 2020: A Bársonyos NATURA 2000 Terület talajfelszíni pókfaunájának felmérése. - Folia Musei historico-naturalis Bakonyiensis 37: 25-35.

Kovícs, P., Szinetár, Cs. \& Eichardt, J. 2009: A I. Magyar Biodiverzitás Napok (Gyürüfü 2006-2008) arachnológiai eredményei (Araneae). - Natura Somogyiensis 13: 43-52.

LoksA, I. 1981: The spider fauna of the Hortobágy National Park (Araneae). - In: MAHunKa S. (ed): The fauna of the Hortobágy National Park. Akadémiai Kiadó, Budapest, 321-339.

MezöFI, L. \& MARKó, V. 2018: Some rare and remarkable spider species from Hungary (Arachnida: Araneae). - Arachnologische Mitteilungen 55: 1-9.

Mikhailov, K. G. \& Szinetár, Cs. 1997: Spiders of the genus Clubiona Latreille, 1804 (Aranei, Clubionidae) in Hungary. - Miscellanea Zoologica Hungarica 11: 49-68.

Nentwig, W., Blick, T., Bosmans, R., Gloor, D., Hänggi, A. \& Kropf C. Spiders of Europe. Version \{no. of month\}. 2021: Online at https://www.araneae.nmbe.ch, accessed on \{date of access 01.03.2021\}. https://doi.org/10.24436/1

SzINETÁR, Cs. 1995: Data to the Araneae fauna of Örség (Western Hungary). - Savaria- Vas Megyei Múzeumok Értesítője 22(2): 245-251.

Szinetár, Cs., Kovács, P. \& EichardT, J. 2015: A kisalföldi meszes homokpuszta katonai használatú gyepterületeinek pókfaunája. - Rence 1: 237-260.

Szinetár, C., Szita, É. \& Kovács, P. 2016: Pókfaunisztikai vizsgálatok a szigligeti Kongó-réten. - Folia Musei Historico-Naturalis Bakonyiensis 33: 75-86. 
